\title{
Quantitative analysis of a rare disease network's international contact database and E-repository provides insights into biobanking in the electronic consent era
}

\author{
Alexander Suarez ${ }^{1 *}$ (D) Curran Reilly ${ }^{2}$ and David C. Fajgenbaum ${ }^{3}$
}

\begin{abstract}
Background: Castleman disease (CD) describes a group of rare and poorly understood lymphoproliferative disorders that include unicentric CD (UCD), Human Herpes Virus-8 (HHV8)-associated multicentric CD (HHV8 + MCD), and HHV8negative/idiopathic MCD (iMCD). Efforts to advance research and drug discovery for CD have been slowed by challenges shared by other rare diseases, such as collecting and centralizing data and biospecimens for research. To collect disease characteristic data and identify individuals interested in contributing biospecimens for research, a global research organization - the Castleman Disease Collaborative Network (CDCN) - established an international Contact Database and electronic repository (E-repository). Herein, we performed analyses of these datasets to further characterize CD and gain insights into research biospecimen acquisition.
\end{abstract}

Results: Descriptive statistical analyses were performed on 891 participants from the Contact Database and 166 patients in the E-repository. The median age of patients at the time of enrollment in the Contact Database and E-repository was $42 \pm 15.7$ and $35 \pm 14.8$, respectively. The E-repository had increased representation from patients with MCD and the iMCD subtype compared to other sub-groups. Though the majority of participants were from the USA, a total of 49 countries on 6 continents were represented. Several patient characteristics in the Contact Database were associated with subsequent enrollment in the E-repository. There were significantly more MCD patients $(p<0.0001)$ and females $(p=$ 0.002) enrolled in the E-repository compared to the Contact Database. Patient's year of birth, date of registration, preferred method of communication, and relationship to the patient were also significantly associated with enrollment in the eRepository.

Conclusions: This study of the largest- dataset of CD patients worldwide provides insights into disease phenotypes, characteristics of patients interested in contributing data and biospecimens for research, and methods for successfully acquiring data and biospecimens. Generally, the factors associated with enrollment in the E-repository represented severity of disease subtype, proximity to the research, and patient motivation. We hope that these findings and the sample documentation (e.g., electronic consent, recruitment materials) provided with this article will assist future rare disease efforts with overcoming hurdles.

Keywords: Castleman disease, Biorepository, Electronic consent, Contact database, Electronic repository, Enrollment strategy

\footnotetext{
* Correspondence: alexanderdanielsuarez@gmail.com

${ }^{1}$ Perelman School of Medicine, University of Pennsylvania, Philadelphia, PA,

USA

Full list of author information is available at the end of the article
}

(c) The Author(s). 2019 Open Access This article is distributed under the terms of the Creative Commons Attribution 4.0 International License (http://creativecommons.org/licenses/by/4.0/), which permits unrestricted use, distribution, and reproduction in any medium, provided you give appropriate credit to the original author(s) and the source, provide a link to the Creative Commons license, and indicate if changes were made. The Creative Commons Public Domain Dedication waiver (http://creativecommons.org/publicdomain/zero/1.0/) applies to the data made available in this article, unless otherwise stated. 


\section{Background}

In the United States, a rare or orphan disease is defined as any disease affecting fewer than 200,000 people [1]. Collectively, 1 in 10 Americans suffer from one of the approximately 7000 rare diseases; only 5\% currently have FDA-approved treatments [2]. Rare disease research faces many challenges that impede discoveries and new drug development, including decreased priority, focus, or funding relative to more common diseases. Due to the nature of rare diseases - few cases worldwide, complicated diagnoses, restricted availability of information and resources - accessing necessary biospecimens and patient data pose additional challenges.

Castleman disease (CD) is a group of rare and poorly understood lymphoproliferative disorders. Clinicians and researchers have primarily relied on case reports to understand CD, as limited access to samples and data has resulted in studies with small numbers. While all cases of CD share common lymph node histopathological features, CD is further sub-classified into: Unicentric CD (UCD), Human Herpesvirus-8 (HHV-8)-associated multicentric CD (HHV8 + MCD), and HHV-8-negative/idiopathic MCD (iMCD) [3]. UCD involves a single region of enlarged lymph nodes and mild symptoms, which can be resolved with lymph node resection. HHV8 + MCD involves multiple regions of enlarged lymph nodes and symptoms related to intense immune hyperactivation due to uncontrolled infection with HHV-8. iMCD is characterized by nearly identical clinical and laboratory abnormalities as HHV8 + MCD, but these patients are HHV-8 negative and the etiology is unknown. Historically iMCD has received the least research attention. Unsurprisingly, it is also the least well understood and most deadly subtype. A review of all published case of MCD was performed in 2016 to further investigate and define iMCD [4]. 42\% of patients were found to be HHV-8positive; 25\% had unknown HHV8 status; and 33\% were HHV-8-negative. This study provided important insights into clinical and pathological features of iMCD, but publication bias could not be accounted for as every case had been previously written up.

In 2012, the Castleman Disease Collaborative Network $(\mathrm{CDCN})$ was created to advance research and treatment discovery for $\mathrm{CD}$. The $\mathrm{CDCN}$ forged a novel framework for research called the collaborative network approach to promote collaboration, prioritize and fund high impact research, facilitate tissue and data sharing for research, and support patients [5]. In order to facilitate tissue and data sharing for research, the $\mathrm{CDCN}$ launched an international Contact Database in July 2014 and an electronic repository (E-repository) in July 2015. The Contact Database collects a limited set of data on patients and loved ones; the E-repository enables patients to indicate their interest in donating tissue samples for research.
Our aim is to characterize the largest dataset of CD patients in the Contact Database and to compare the features of patients who also sign up for the E-repository against those who do not. We also share resources, including a patient-directed electronic consent (e-consent) and marketing materials, that can be re-used by other rare disease efforts that are interested in improving research biospecimen acquisition.

\section{Methods \\ Patient databases}

The CDCN webpages for participants to sign up for the Contact Database and the E-repository are shown in Additional file 1: A. The Contact Database includes an online questionnaire regarding participant demographic data, diagnosis, contact information, and preferences for getting more involved. Following completion of the Contact Database form, the participant receives an automated email that includes a link to sign up for the Erepository, among other links (Additional file 1: B1-B2). Within $24 \mathrm{~h}$, the participant also receives a personalized follow-up email from a $\mathrm{CDCN}$ staff member, which also includes a link to sign up for the E-repository.

The E-repository includes a Tissue Sample Donation Interest Form (TSDIF) to collect more in depth information regarding the patient's demographics, course of disease, past medical history, and any previous tissue donation (See Additional file 1: C for full list of questions). Completion of this form to express interest in participating in research is included as part of a protocol ("The Castleman Disease Collaborative Network Biobank: A Collection of Biospecimens and Clinical Data to Facilitate Research") approved by Quorum IRB. A separate electronic consent form was developed for the CDCN's Biobank (see Additional file 1: D). Participants can request to be removed from the Contact Database and E-repository at any time.

\section{Statistical analyses}

Descriptive statistical analyses were performed on the demographic and disease characteristic data for both databases. Not all patients completed each registration question for the Contact Database and E-repository form. Chi-square analysis was performed to search across the two databases for significant associations between characteristics of patients in the Contact Database and likelihood of joining the E-repository. The Bonferroni method of multiple hypothesis testing was performed to determine corrected $p$-values. Alpha was determined to be $p$-value $<0.05$.

\section{Results}

The Contact Database includes 891 patients enrolled between July 2014-December 2017, and the E-repository 
includes 166 patients enrolled between July 2015-December 2017. There are 165 patients in both the Contact Database and the E-repository; one patient is in the Erepository but not the Contact Database. Table 1 includes demographics of patients in the Contact Database and E-repository. Of those participants registered in the Contact Database $(n=891), 18.2 \%$ also registered for the E-repository $(n=165)$. In the Contact Database, $49 \%$ of patients were female and $51 \%$ of patients were male; $72 \%$ of patients were from the US. The remaining $28 \%$ of participants represent 49 countries on 6 continents (Fig. 1). The mean age of patients at the time of enrollment was 41.8 years old. The proportion of patients with CD subtypes included 41\% MCD, 33\% UCD, and 26\% unknown. Sixty percent of MCD patients were female and $69 \%$ of UCD patients were female (Table 2). Among MCD cases, $57 \%$ were reported to be HHV-8-negative/ iMCD, 10\% HHV8 + MCD, and 33\% unknown.

Patients in the E-repository were $67 \%$ female and $82 \%$ were from the US. The mean age of patients was 42.6 years old. Fifty-one percent of patients reported having MCD compared to $42 \%$ UCD and $7 \%$ unknown. Sixtyfour percent of MCD cases reported having HHV-8negative/iMCD, $8 \%$ HHV8 + MCD, and 27\% unknown.

In order to identify features significantly associated with enrollment in the E-repository, differences in the characteristics of patients in the Contact Database and Erepository were evaluated (Table 3). The distribution of patient gender and primary diagnosis was significantly different between patients in the Contact Database and Erepository (corrected $P<0.05$ after correcting for multiple hypothesis testing). Additional characteristics significantly associated with enrollment in the E-repository included patient's year of birth, date of registration, preferred method of communication, and participant's relationship to the patient. Elements that trended towards significance included patient's country of origin, the participant's interest in joining a patient engagement program, and the amount of time elapsed between diagnosis and date of enrollment. There were no differences in patients' subtype of MCD, age at diagnosis, date of diagnosis, and disease specific symptoms between the two cohorts.

The demographics of those who elected to register for both the Contact Database and E-repository are summarized in Table 4. Among those who signed up for the Contact Database, women had nearly a two-fold increased rate of joining the E-repository when compared to men (22.3 vs. 10.6\%). Patients born either between 1961 and 1970 (27.1\%) or 2011-2018 (27.1\%), compared to those patients of other age cohorts, were also significantly more likely to enroll. MCD patients $(28.6 \%)$ and UCD patients $(29.2 \%)$ were significantly more likely to enroll in the E-repository than participants who were unsure of diagnosis or not officially diagnosed (6.1\%). Date of registration for the Contact Database was also significantly associated with enrollment in the Erepository. Patients who registered for the Contact Database between July-December 2014 (25.9\%), July-

Table 1 Demographic data for patients enrolled in the Contact Database and E-repository, and prior estimates in literature

\begin{tabular}{|c|c|c|c|c|c|}
\hline Title & Contact Database & E-repository & $p$-value* & Talat \& Schulte & Liu et al. \\
\hline $\mathrm{N}$ & 891 & 166 & & 384 & $128 \mathrm{iMCD}$ \\
\hline \multicolumn{6}{|l|}{ Age (Median \pm SD) } \\
\hline UCD & $35 \pm 14.8$ & - & & 30 & - \\
\hline MCD & $42 \pm 15.7$ & - & $0.612^{1}$ & 52 & 50 \\
\hline Patient Gender, N (\%) & & & $0.002^{2}$ & & \\
\hline Female & $200(49 \%)$ & $44(67 \%)$ & & $179(47 \%)$ & $54(42 \%)$ \\
\hline Male & 195 (51\%) & $22(33 \%)$ & & $205(53 \%)$ & $74(58 \%)$ \\
\hline Patient Country of Origin, N (\%) & & & $0.002^{2}$ & & \\
\hline US & $469(72 \%)$ & 117 (82\%) & & - & - \\
\hline International & $185(28 \%)$ & $26(18 \%)$ & & - & - \\
\hline Primary Diagnosis, N (\%) & & & $<0.0001^{2}$ & & \\
\hline Multicentric CD & $206(41 \%)$ & $59(51 \%)$ & & $101(26 \%)$ & 128 \\
\hline Unicentric CD & $168(33 \%)$ & 49 (42\%) & & $283(74 \%)$ & - \\
\hline Unknown & $131(26 \%)$ & $8(7 \%)$ & & - & - \\
\hline Patient's MCD Subtype Diagnosis, N (\%) & & & $0.403^{2}$ & & \\
\hline HHV-8-negative & $117(57 \%)$ & $38(64 \%)$ & & - & 128 \\
\hline HHV-8-positive & $21(10 \%)$ & $5(8 \%)$ & & - & - \\
\hline Not sure or N/A & 67 (33\%) & $16(27 \%)$ & & - & - \\
\hline
\end{tabular}

${ }^{1}$ Student's t-test $p$-value ${ }^{2}$ Chi square $p$-value ${ }^{*} p$-value with Bonferroni correction $(\mathrm{k}=21), \mathrm{a}=.002$ 


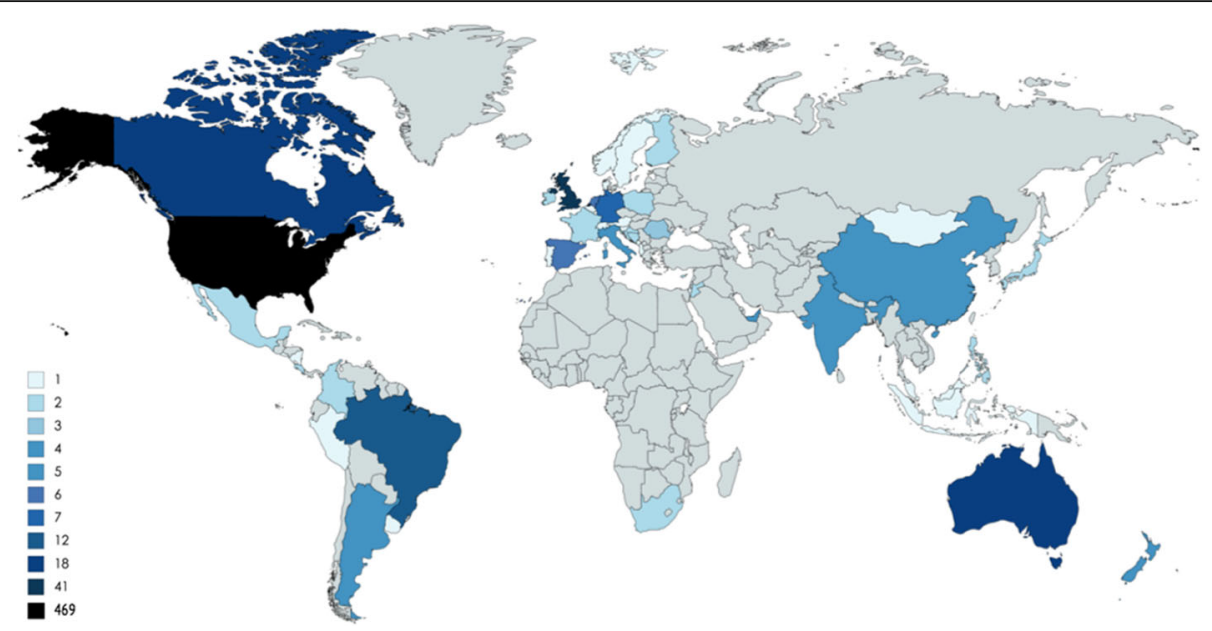

Fig. 1 Distribution of Patients in Contact Database by Country $(n=654)$

December 2015 (30.4\%), and January-June 2016 (25.4\%) were more likely to sign up for the E-repository than registrants at other time periods. Of the patients who listed both phone and email as their preferred methods of communication (rather than just one or neither), $100 \%$ signed up for the E-repository. When patients registered themselves directly for the Contact Database, they were more likely to also register for the Erepository (27.8\%), compared to those patients who were registered by family $(4.1 \%)$ or friends $(3.2 \%)$.

We hypothesized that there would be a statistically significant rise in enrollment in the Contact Database and E-repository following the 2017 Patient Symposium, but there was no significant change. Figure 2 illustrates patient enrollments over time in both the Contact Database and the E-repository through December 2017. The Contact Database followed a steady and linear enrollment trajectory from its opening, averaging about 21 participants a month. For the E-repository, few individuals initially signed up ( 8 individuals in the first 8 months). Upon execution of a comprehensive enrollment strategy in March 2016 (see Additional file 1: E), there was a large rise in enrollment. Thirty-four patients signed up within 1 week and 56 signed up within 1 month. Over the next several months, the rate of enrollment continued at an increased pace and by the end of 2016 there were 134 patients in the E-repository.

Table 2 Patient-reported subtype of CD by gender in the Contact Database

\begin{tabular}{lll}
\hline Title & Male & Female \\
\hline Primary Diagnosis, N (\%) & & \\
Multicentric CD & $81(40 \%)$ & $120(60 \%)$ \\
Unicentric CD & $38(31 \%)$ & $86(69 \%)$ \\
\hline
\end{tabular}

\section{Discussion}

Herein we present a description of the largest database of CD patients worldwide, characteristics associated with patients interested in contributing data and biospecimens for research, and insights into successful data and biospecimen acquisition for rare diseases.

The characteristics of $\mathrm{CD}$ patients in the Contact Database share several commonalities and differences with the existing literature. The age $(41.8 \pm 16.0)$ of $C D$ patients in the Contact Database is similar to what has been reported previously for $\mathrm{CD}$ patients by Munshi et al. (mean: 42.9-45.8, SD 17.2-17.6) [6]. For subtypes of $\mathrm{CD}$, the data also aligns with prior published ages from Talat \& Schulte (median: 30 and 52 for UCD and MCD respectively) [7] and iMCD patients from Liu et al. (median: 50, IQR 35-61) [4]. The gender distribution (49\% female) is also similar to that published by Munshi et al. (64.1\%) for CD and Liu et al. (42.2\%) for iMCD. Review articles often state that UCD predominantly affects females while MCD affects more males [8, 9]. However, when broken down by gender, both UCD and MCD had more females than males. There did seem to be a larger female predominance in the UCD group as compared to the MCD group, in line with the UCD-female association canon.

The only study of the incidence of $\mathrm{CD}$ estimated that MCD cases made up 1569-1756 (20-27\%) of the 65027696 annual CD diagnoses in the USA [6]. Our results (41\% MCD, 33\% UCD, 26\% unknown) suggest that the proportion of $\mathrm{CD}$ cases that have MCD may be higher than previous estimates. However, the Contact Database is highly susceptible to ascertainment bias and likely also does not represent the population distribution, as individuals choose to join the Contact Database for a variety of reasons. Factors such as personal motivation, access to the internet, technical savviness, and ability to 
Table 3 Summary of comparison of characteristics of patients in the Contact Database and E-Repository by Chi Square, displayed in order by $p$-value

\begin{tabular}{|c|c|}
\hline Title & $\begin{array}{l}p^{-} \\
\text {value* }\end{array}$ \\
\hline \multicolumn{2}{|l|}{ Statistically significant $(a<0.002)$} \\
\hline Patient's Primary Diagnosis & $\begin{array}{l}< \\
0.0001\end{array}$ \\
\hline Date of Registration & $\begin{array}{l}< \\
0.0001\end{array}$ \\
\hline Participant's Preferred Method of Communication & $\begin{array}{l}< \\
0.0001\end{array}$ \\
\hline Participant's Relationship to Patient & $\begin{array}{l}< \\
0.0001\end{array}$ \\
\hline Patient's Year of Birth & 0.001 \\
\hline Patient's Gender & 0.002 \\
\hline \multicolumn{2}{|l|}{ Trending towards significance $(a=0.002-0.01)$} \\
\hline Patient's Country & 0.002 \\
\hline $\begin{array}{l}\text { Time elapsed between date of diagnosis and date of } \\
\text { registration }\end{array}$ & 0.005 \\
\hline Interest in Warrior program & 0.007 \\
\hline \multicolumn{2}{|l|}{ Not statistically significant $(a>0.01)$} \\
\hline Patient's Date of Diagnosis & 0.075 \\
\hline Patient's Symptoms & 0.177 \\
\hline Patient's Subtype Diagnosis & 0.403 \\
\hline Patient's Age at Diagnosis & 0.499 \\
\hline Participant's Age & 0.502 \\
\hline Participant's Gender & 0.615 \\
\hline Participant's Year of Birth & 0.721 \\
\hline $\begin{array}{l}\text { Distance between participant and patient based on zip } \\
\text { code }\end{array}$ & 0.742 \\
\hline Registration Date relative to annual Patient Summit & 0.851 \\
\hline
\end{tabular}

navigate an English-language website may have contributed. Further, after surgical excision and treatment of their disease, UCD patients may proceed with their healthy lives and not be as interested in volunteering in research studies for their already treated disease as patients with MCD. The only prior estimate of the proportion of $\mathrm{HHV} 8$-negative iMCD relative to $\mathrm{HHV} 8+\mathrm{MCD}$ was $34-55 \%$ vs $45-66 \%$, respectively, based on the proportion in published case reports [4]. Our results (57$90 \%$ iMCD vs $10-43 \%$ HHV8 + MCD) suggest that the proportion of iMCD to HHV8 + MCD may be higher than previously thought.. However, patients with iMCD, which is not as clearly understood or well controlled as HHV8 + MCD, may be more likely to seek out support and answers through a rare disease organization as well as more likely to express interest in donating biospecimens for research. Likewise, the high proportion of patients enrolled from the US almost certainly represents the fact that the $\mathrm{CDCN}$ is headquartered in the US and media profiles have primarily occurred within the US.

The high proportion of respondents unaware of whether they had UCD or MCD is quite concerning as the clinical presentation, treatment, and survival outlook is significantly different. Approximately 35\% of iMCD patients die within 5 years of diagnosis and another $25 \%$ die within 10 years of diagnosis [10]. Conversely, less than $5 \%$ of UCD patients die within 5 years of diagnosis and less than $10 \%$ of HHV8 + MCD patients die if appropriately treated with $\mathrm{B}$ cell depletion therapy. It is imperative that all physicians perform necessary testing to determine if the patient has UCD, HHV $8+\mathrm{MCD}$, or iMCD and that patients are better informed of their subtype. A possible explanation for this high proportion of patients unaware of their subtype may be that some individuals who register in the Contact Database don't actually have $\mathrm{CD}$ or the subtype testing may not have been completed when the patient registered. A clear limitation of this study is that the patients' diagnoses are patient reported and not validated with medical records. There may be discrepancies between what participants complete in the form and the medical record. The CDCN is currently co-leading enrollment into the ACCELERATE Natural History Registry, where patients can enroll themselves online, complete medical surveys, and grant access to medical records for in-depth data abstraction. By coupling selfreported surveys with medical record data, we should be able to gain insights into the reliability of self-reported surveys like the Contact Database and E-repository. The recent publication of the first-ever diagnostic criteria for iMCD may also help with determining and communicating this challenging diagnosis appropriately [11].

The analysis of differences between patients who only enroll in the Contact Database versus those who go on to enroll in the E-repository suggests that certain characteristics are associated with interest in tissue sample donation, such as female gender and MCD subtype. Female gender has previously been shown to be associated with more altruistic behavior such as filling out more surveys and donating money to nonprofits [12, 13]. Diagnosis with the more severe MCD subtype compared to UCD or unknown subtype of CD was also associated with significantly increased rates of E-repository enrollment. Some of the respondents with unknown subtype of CD may not be confident they actually have $\mathrm{CD}$ and so therefore may be less interested to contribute samples to its cause. Another explanation is that those patients who have a known primary diagnosis communicated to them are more motivated to participate in investigative efforts. The significant differences between the characteristics of patients in the Contact Database and the E-repository, including female gender, proximity to research/USA location, and more severe subtype may 
Table 4 Odds Ratios of joining E-repository for Significant Characteristics

\begin{tabular}{|c|c|c|c|c|}
\hline Title & Joined E repository & Did not join & $\begin{array}{l}\text { Rate of } \\
\text { E-repository }\end{array}$ & Odds Ratio ${ }^{a}$ \\
\hline Patient's Gender & $n=395$ & & & \\
\hline Male & 22 & 178 & $11.0 \%$ & 0.60 \\
\hline Female & 44 & 151 & $22.6 \%$ & 1.24 \\
\hline Patient's Year of Birth & $n=721$ & & & \\
\hline 1930-1940 & 0 & 12 & $0.0 \%$ & 0.00 \\
\hline $1941-1950$ & 9 & 25 & $26.5 \%$ & 1.45 \\
\hline $1951-1960$ & 16 & 84 & $16.0 \%$ & 0.88 \\
\hline $1961-1970$ & 48 & 127 & $27.4 \%$ & 1.51 \\
\hline 1971-1980 & 27 & 124 & $17.9 \%$ & 0.98 \\
\hline 1981-1990 & 34 & 100 & $25.4 \%$ & 1.39 \\
\hline 1991-2000 & 5 & 74 & $6.3 \%$ & 0.35 \\
\hline $2001-2010$ & 2 & 19 & $9.5 \%$ & 0.52 \\
\hline 2011-2018 & 5 & 10 & $33.3 \%$ & 1.83 \\
\hline Patient's Primary Diagnosis & $n=505$ & & & \\
\hline Multicentric CD & 59 & 147 & $28.6 \%$ & 1.57 \\
\hline Unicentric CD & 49 & 119 & $29.2 \%$ & 1.60 \\
\hline Unsure or Not officially diagnosed & 8 & 123 & $6.1 \%$ & 0.34 \\
\hline Date of Registration & $n=874$ & & & \\
\hline Jul-Dec 2014 & 30 & 86 & $25.9 \%$ & 1.42 \\
\hline Jan-Jun 2015 & 26 & 113 & $18.7 \%$ & 1.03 \\
\hline Jul-Dec 2015 & 31 & 71 & $30.4 \%$ & 1.67 \\
\hline Jan-Jun 2016 & 35 & 103 & $25.4 \%$ & 1.39 \\
\hline Jul-Dec 2016 & 17 & 87 & $16.3 \%$ & 0.90 \\
\hline Jan-Jun 2017 & 7 & 138 & $4.8 \%$ & 0.27 \\
\hline Jul-Dec 2017 & 6 & 124 & $4.6 \%$ & 0.25 \\
\hline Participant's Preferred Method of Communication & $n=449$ & & & \\
\hline Email & 66 & 340 & $16.3 \%$ & 0.89 \\
\hline Phone & 7 & 25 & $21.9 \%$ & 1.20 \\
\hline Phone, email & 11 & 0 & $100.0 \%$ & 5.49 \\
\hline Participant's Relationship to Patient & $n=811$ & & & \\
\hline Patient & 150 & 380 & $28.3 \%$ & 1.55 \\
\hline Family & 9 & 209 & $4.1 \%$ & 0.23 \\
\hline Friends & 2 & 61 & $3.2 \%$ & 0.17 \\
\hline
\end{tabular}

${ }^{\mathrm{a} O v e r a l l ~ a v e r a g e ~} 18.2 \%$

also be associated with tissue sample donation interest for other rare diseases too.

Additional insights were generated related to patient interest in contributing samples for research. Participants that A) signed up within the first 2 years of the Contact Database opening, B) preferred to be contacted both by email or phone (rather than one or neither), or $\mathrm{C}$ ) were the patient (rather than a family member) were all significantly more likely to enroll in the E-repository. Regarding A, it seems that there was a first mover effect. Those individuals who were quick to sign up in the Contact Database were also more willing to express interest in donating tissue; these individuals were driven by the desire to be as involved as much as possible as quickly as possible. Similarly, even though a small number of individuals elected to be reached out to by both phone and email $(n=11)$, all eleven individuals signed up for the E-repository. As would be expected, individuals open to any form of contact regardless of the modality likely have a stronger desire to participate in research efforts.

Three characteristics that trended towards significance are also worth discussing. American participants trended towards being more likely to sign up for the E- 


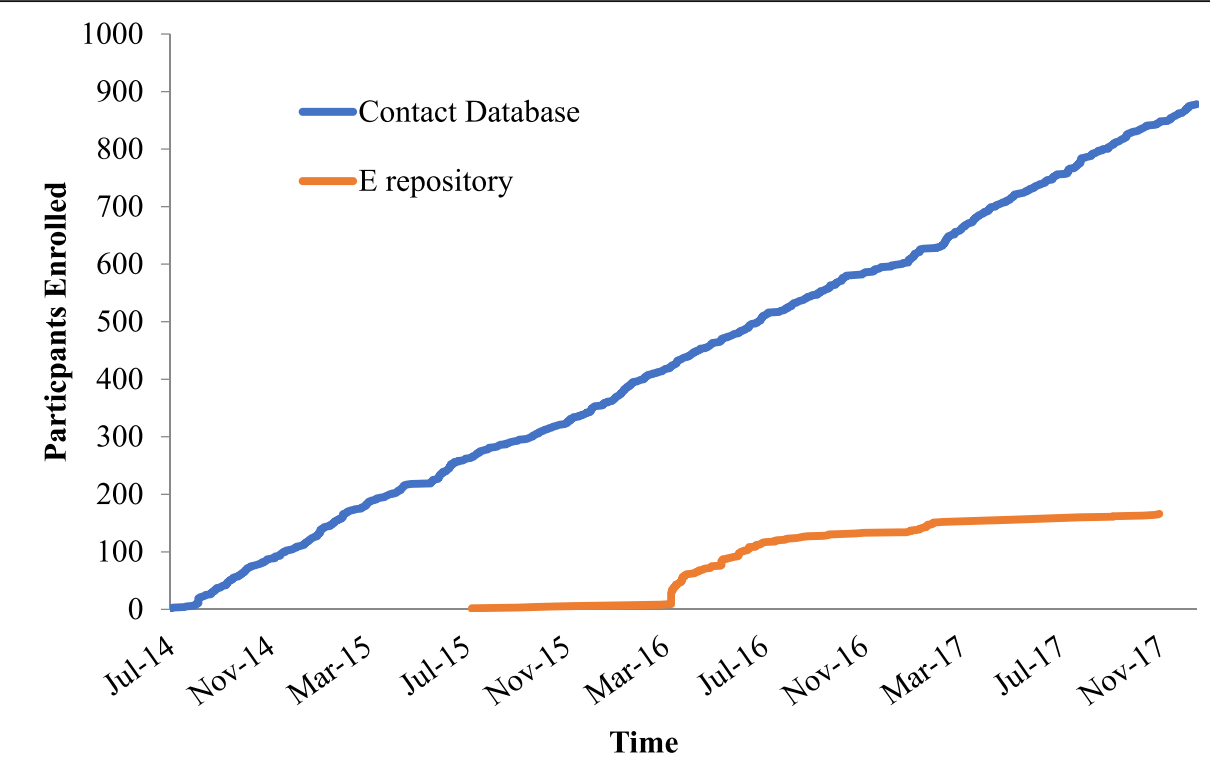

* "Contact Database and E-repository launched on July 2014 and July 2015,

respectively."

Fig. 2 Graph of participant enrollment in the Contact Database and E-repository from July 2014 until December 2017. * "Contact Database and Erepository launched on July 2014 and July 2015, respectively"

repository. This likely has to do with the CDCN's location in the USA and the proximity to most CDCN research studies. Individuals who expressed interest in a CDCN program geared at empowering patients and loved ones to spread awareness and raise funds for research also tended to be more likely to enroll in the Erepository. We suspect similar underlying motivations in these patients as those outlined for individuals who answered phone and email for their preferred communication. Finally, individuals who had carried a CD diagnosis for a longer period of time also trended towards being more likely to express interest in donating tissue. This may be secondary to these individuals experiencing a prolonged course with little scientific progress, which may have served as inspiration to get more involved with research. This may also reflect that patients further out from diagnosis are able to consider contributing to research more so than newly diagnosed patients, who are focused on finding an expert and getting care right away.

There are also several operational learnings from implementing and rolling out the Contact Database and E-repository, which may be helpful for other rare disease organizations. First, an electronic consent form has been shown to be effective for increasing enrollment into tissue repositories for a number of disease-specific and non-disease specific biobanks [14]. The IRB-approved electronic consent forms that the $\mathrm{CDCN}$ developed are included as supplementary files with this publication so other rare disease organizations may model their own consents after it in hopes of expediting other rare disease investigative efforts (Additional file 1: D). Second, visualizing the E-repository enrollment (Fig. 2) highlights a sharp peak around March 2016. A review of the CDCN's internal records helps to explain the spike in enrollment. A CDCN task force developed a tissue acquisition plan in February 2016 called "The Answers are Within." Only 8 patients had been enrolled in the Erepository in the prior 8 months. The strategy included posting an article to the homepage on CDCN.org, posting on social media, and sending tailored emails (Additional file 1: E) to members of the Contact Database. There were different pathways of communication for patients who reported having $\mathrm{UCD}, \mathrm{MCD}$, or being unsure. The outcome was a 15 -fold increase ( 8 vs. 125 patients) in enrollment when comparing the 8 months pre vs post roll out of the strategy. Finally, considering the low enrollment of patients in Asian and African countries, moving forward, a greater emphasis should be made to partner with physicians in these regions and to adapt English language consents to local languages to encourage more patient enrollments in these regions. As larger numbers are enrolled into these databases within a given country, analyses should be conducted to determine regional differences in prevalence and demographics at the national level.

Despite several important limitations, the results presented come from the largest database of $\mathrm{CD}$ patients. 
The characterization of patients in the Contact Database and comparison with patients in the E-repository identified statistically significant differences that may assist with future rare disease biospecimen procurement. Commentary on managing these databases and sample documentation (e.g., electronic consent, recruitment materials) provided with this article should also assist future rare disease research efforts with adoption and implementation of biorepositories.

\section{Conclusions}

This study of the largest- dataset of CD patients worldwide provides insights into disease phenotypes, characteristics of patients interested in contributing data and biospecimens for research, and methods for successfully acquiring data and biospecimens. Generally, the factors associated with enrollment in the E-repository represented severity of disease subtype, proximity to the research, and patient motivation. We hope that these findings and the sample documentation (e.g., electronic consent, recruitment materials) provided with this article will assist future rare disease efforts with overcoming hurdles.

\section{Additional file}

Additional file 1: Examples of webpage screenshots, email communication, tissue donation forms, electronic consents, and Erepository enrollment documents. (PDF 1553 kb)

\section{Acknowledgements}

We would like to acknowledge all those individuals at the Castleman Disease Collaborative Network for their important support of this project.

\begin{abstract}
Authors' contributions
AS made substantial contributions to the conception, design of the work; the acquisition, analysis, interpretation of data, as well as the draft the work and subsequent revisions. CR made substantial contributions to the conception, design of the work; the acquisition, analysis, interpretation of data, as well as the draft the work and subsequent revisions. DF made substantial contributions to the conception, design of the work; the acquisition, analysis, interpretation of data, as well as the draft the work and subsequent revisions. All authors read and approved the final manuscript.
\end{abstract}

\section{Funding}

The funding for the project came from the Castleman Disease Collaborative Network.

\section{Availability of data and materials}

The data that support the findings of this study are available from the authors upon reasonable request and with permission of Quorum IRB. Much of the data infrastructure has been made available in our Additional file 1.

\section{Ethics approval and consent to participate}

"The Castleman Disease Collaborative Network Biobank: A Collection of Biospecimens and Clinical Data to Facilitate Research" was approved by Quorum IRB (IRB 00003226).

\section{Consent for publication}

Not applicable.

\section{Competing interests}

The authors declare that they have no competing interests.

\section{Author details}

${ }^{1}$ Perelman School of Medicine, University of Pennsylvania, Philadelphia, PA USA. ${ }^{2}$ Castleman Disease Collaborative Network, Philadelphia, PA, USA.

${ }^{3}$ Division of Translational Medicine and Human Genetics, Perelman School of Medicine, University of Pennsylvania, Philadelphia, PA, USA.

Received: 13 February 2019 Accepted: 25 June 2019

Published online: 11 July 2019

\section{References}

1. Public Law 97-414: To amend the Federal Food, Drug, and Cosmetic Act to facilitate the development of drugs for rare diseases and conditions and for other purposes. [Orphan Drug Act]" (96 Stat. 2049; Date: 1/4/83). Text from: United States Public Laws; 1983. Accessed 05 July 2019.

2. Sardana D, Zhu C, Zhang M, Gudivada RC, Yang L, Jegga AG. Drug repositioning for orphan diseases. Brief Bioinform. 2011;12(4):346-56.

3. Fajgenbaum DC, van Rhee F, Nabel CS. HHV-8-negative, idiopathic multicentric Castleman disease: novel insights into biology, pathogenesis, and therapy. Blood. 2014;123(19):2924-33.

4. Liu AY, Nabel CS, Finkelman BS, Ruth JR, Kurzrock R, van Rhee F, Krymskaya VP, Kelleher D, Rubenstein AH, Fajgenbaum DC. Idiopathic multicentric Castleman's disease: a systematic literature review. Lancet Haematol. 2016;3(4):e163-75.

5. Fajgenbaum DC, Ruth JR, Kelleher D, Rubenstein AH. The collaborative network approach: a new framework to accelerate Castleman's disease and other rare disease research. Lancet Haematol. 2016;3(4):e150-2.

6. Munshi N, Mehra M, van de Velde H, Desai A, Potluri R, Vermeulen J. Use of a claims database to characterize and estimate the incidence rate for Castleman disease. Leuk Lymphoma. 2015;56(5):1252-60.

7. Talat N, Schulte KM. Castleman's disease: systematic analysis of 416 patients from the literature. Oncologist. 2011. https://doi.org/10.1634/ theoncologist.2011-0075.

8. Mylona EE, Baraboutis IG, Lekakis LJ, Georgiou O, Papastamopoulos V, Skoutelis A. Multicentric Castleman's disease in HIV infection: a systematic review of the literature. AIDS Rev. 2008;10(1):25-35.

9. Marcin J. "Castleman Disease: What Is It and How is it Treated?" Healthline; 2017.

10. Dispenzieri A, Armitage JO, Loe MJ, Geyer SM, Allred J, Camoriano JK, Menke DM, Weisenburger DD, Ristow K, Dogan A, Habermann TM. The clinical spectrum of Castleman's disease. Am J Hematol. 2012;87(11):997-1002.

11. Fajgenbaum DC, Uldrick TS, Bagg A, Frank D, Wu D, Srkalovic G, Simpson D, Liu AY, Menke D, Chandrakasan S, Lechowicz MJ. International, evidence-based consensus diagnostic criteria for HHV-8negative/idiopathic multicentric Castleman disease. Blood. 2017. https:// doi.org/10.1182/blood-2016-10-746933.

12. Curtin $R$, Presser $S$, Singer $E$. The effects of response rate changes on the index of consumer sentiment. Public Opin Q. 2000;64(4):413-28.

13. Rooney PM, Mesch DJ, Chin W, Steinberg KS. The effects of race, gender, and survey methodologies on giving in the US. Econ Lett. 2005:86(2):173-80.

14. Boutin NT, Mathieu K, Hoffnagle AG, Allen NL, Castro VM, Morash M, O'Rourke PP, Hohmann EL, Herring N, Bry L, Slaugenhaupt SA. Implementation of electronic consent at a biobank: an opportunity for precision medicine research. J Pers Med. 2016;6(2):17.

\section{Publisher's Note}

Springer Nature remains neutral with regard to jurisdictional claims in published maps and institutional affiliations.

Ready to submit your research? Choose BMC and benefit from:

- fast, convenient online submission

- thorough peer review by experienced researchers in your field

- rapid publication on acceptance

- support for research data, including large and complex data types

- gold Open Access which fosters wider collaboration and increased citations

- maximum visibility for your research: over $100 \mathrm{M}$ website views per year

At BMC, research is always in progress.

Learn more biomedcentral.com/submission 